\title{
HSUMB Newsletter
}

Hellenic Society for Ultrasound in Medicine and Biology

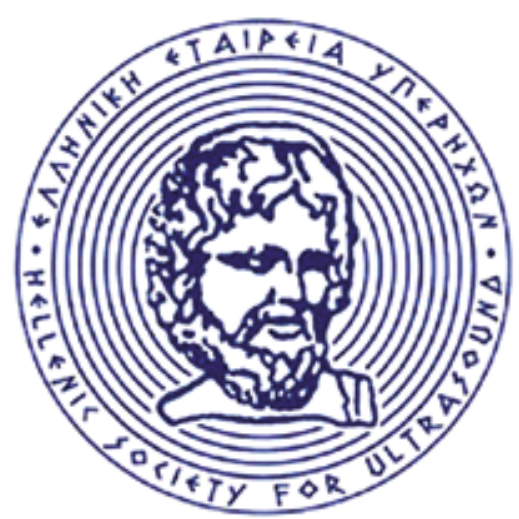

HSUMB Educational Program of 2016

HSUMB participated and organized an Educational Ultrasound Seminar in cooperation with 2 National Congress during the first half of the year 2016. The first took place on May 12, 2016 during the $42^{\text {nd }}$ Panhellenic Yearly Medical Congress. There were 34 participants, mainly general physicians who were introduced to the basic rules of ultrasound and had the opportunity to have handson experience under the guidance of our specialized instructors
The second seminar was help within the context of the 22nd Scientific Congress of Medical Students and New Doctors in Greece. Participants were 30. Brief presentations of Ultrasound Applications in the Upper and Lower Abdomen, Thyroid and Breast and most importantly FAST protocols and Emergency US were followed by hands-on training workshops where the students and new doctors had their first ultrasound scanning experience.

\section{Address}

Nikolaos Papantoniou MD PhD

Vas. Sofias 82

Athens, Greece info@hsumb.gr 